\title{
Determinación de la profundidad de calado y velocidad de hundimiento en redes de cerco utilizadas en el norte de Chile
}

\author{
Teófilo Melo, Alex Martínez, Dante Queirolo \& Carlos Hurtado \\ Escuela de Ciencias del Mar, Pontificia Universidad Católica de Valparaíso, Casilla 1020, Valparaíso \\ E-mail: tecpes@ucv.cl
}

\begin{abstract}
RESUMEN. En septiembre de 2000 y enero de 2001 se efectuaron experiencias dirigidas a determinar la profundidad de calado y velocidad de hundimiento de redes de cerco empleadas en la pesquería de pequeños pelágicos de la zona norte de Chile. Durante este período, se efectuaron 10 salidas de pesca en dos embarcaciones características de la flota, con un total de 21 lances. En cada embarcación se emplearon redes de similares dimensiones y características de diseño. Sin embargo, ambas redes presentaron diferencias en la construcción de los cuerpos centrales, con dos tipos de tela; con nudo y sin nudo. Se comparó la profundidad máxima de calado de las redes y el tiempo necesario para alcanzar dicha profundidad mediante el uso de batiquimógrafos, determinándose la velocidad media de hundimiento durante cada lance de pesca. Además, se estudió la profundidad alcanzada por la red durante el calado en diferenciales de tiempo de $30 \mathrm{~s}$. La profundidad máxima de calado de la red con nudo fue mayor que en la red sin nudo, alcanzando la primera 93,8 m de profundidad, mientras que la segunda sólo 71,3 m. Este mismo comportamiento se observó en la velocidad de hundimiento del cuerpo central, donde la red con nudo fue superior a la red sin nudo. Sin embargo, no se observó diferencia en la velocidad de hundimiento en los cuerpos del copo.
\end{abstract}

Palabras claves: red de cerco, profundidad de calado, velocidad de hundimiento, batiquimógrafo, pesquería pelágica, Chile.

\section{Determination of pursing depth and sinking speed in northern Chilean purse seines}

\begin{abstract}
In September 2000 and January 2001 several experiments were conducted to determine the pursing depth and sinking speed in purse seines employed in the small pelagic fishery in the northern of Chile. During this period, 10 fishing trips were performed on two of the most common type of vessels, with a total of 21 purse seine sets. Similar dimensions and characteristic design nets were employed for both fishing vessels in this research. Nevertheless, both nets showed different constructions with two kinds of netting central bodies; knots and knotless. The maximum pursing depth and the required time to reach them were compared by means of bathykymograph measures, and the average sinking speed for each set was determinate as well. Furthermore, the pursing depths were analyzed through a protracted series of observations each $30 \mathrm{~s}$ apart during the fishing sets. The maximum average pursing depth of the knot's netting was higher than the knotless one, such that the knot netting purse seine reached 93,8 $\mathrm{m}$ in depth and the knotless netting purse seine reached only $71,3 \mathrm{~m}$. The same statistical behaviour was observed in the sinking speed of the central body, were the knot netting was higher than the knotless one. However, no differences were noticed in the bunt net speeds.
\end{abstract}

Key words: purse seine, pursing depth, sinking speed, bathykymograph, pelagic fishery, Chile.

\section{INTRODUCCIÓN}

Las primeras aproximaciones tecnológicas para el estudio del comportamiento de redes de cerco comercial fueron llevadas a cabo por científicos de Japón, URSS y EEUU, quienes desarrollaron ins- trumentación submarina para registrar la profundidad con respecto al tiempo (Hester et al., 1972). Estos instrumentos, llamados batiquimógrafos, fueron usados para estudiar el desempeño de hundimiento de la relinga inferior de una red atunera noruega modificada, en comparación con una red 
híbrida de cerco atunera, demostrando que el diseño de la red presenta una influencia predominante sobre el desempeño en condición de pesca (BenYami, 1994).

La importancia de los estudios con batiquimógrafos radica en la necesidad de detectar un problema que afectó durante años a los pescadores: el escape de los cardúmenes desde las redes. Además, estos estudios han ayudado a medir la velocidad de hundimiento de las diferentes secciones de la relinga inferior en una configuración vertical durante la distintas etapas del proceso de captura (Green, 1969; Prado \& Beirnaert, 1978). Esto hace posible establecer la influencia del diseño de los equipos, del tipo de material de construcción utilizado, de las tácticas empleadas por el capitán de la embarcación durante el calado y la maniobra con relación a la forma de pescar de la red (Ben-Yami, 1994).

Es imprescindible, en consecuencia, conocer la profundidad de calado de éstas, de manera de mejorar las características de construcción y prever las opciones de cambio en caso de modificarse el comportamiento en profundidad del recurso (Ben-Yami, 1994). En este contexto, el objetivo del presente estudio consistió en determinar la profundidad de calado y velocidad de hundimiento en redes de cerco utilizadas en la zona norte de Chile, registrando in situ el desempeño de la red durante el proceso de captura.

\section{MATERIALES Y MÉTODOS}

El estudio se efectuó en septiembre de 2000 y enero de 2001 en el puerto de Mejillones ( $23^{\circ} \mathrm{S}$ ), a bordo de dos embarcaciones cerqueras de alta mar de plantilla americana de $33 \mathrm{~m}$ de eslora y $330 \mathrm{~m}^{3}$ de capacidad de bodega.

\section{Análisis estático}

Las redes utilizadas poseen un total de 14 cuerpos, los cuales se encuentran divididos en tres secciones; la primera, denominada cuerpos de proa, presenta 3 cuerpos con corte; la segunda, denominada cuerpos centrales, posee 10 cuerpos, dentro de los cuales se distingue un cuerpo que posee titulación mayor, también denominado copo centro, que se utiliza como refuerzo durante el lance; y la tercera, denominada cuerpo de popa o colero, con un cuerpo de similares características que los de proa (Fig.1).

En primer lugar, se realizó el análisis estático de las redes anchoveteras empleadas por ambas naves para conocer su diseño, armado y construcción, para lo cual se identificó la Red 1 a aquella construida con tela torcida sin nudo en sus cuerpos centrales y tela torcida con nudo en los cuerpos restantes de proa y popa, y la Red 2 aquella construida completamente con tela torcida con nudo en sus cuerpos de proa, centrales y de popa (Tabla 1). En cuanto a las relingas de ambas redes, tanto la superior como inferior, están constituidas por el mismo tipo de material, construcción y diámetro (Tabla 2).

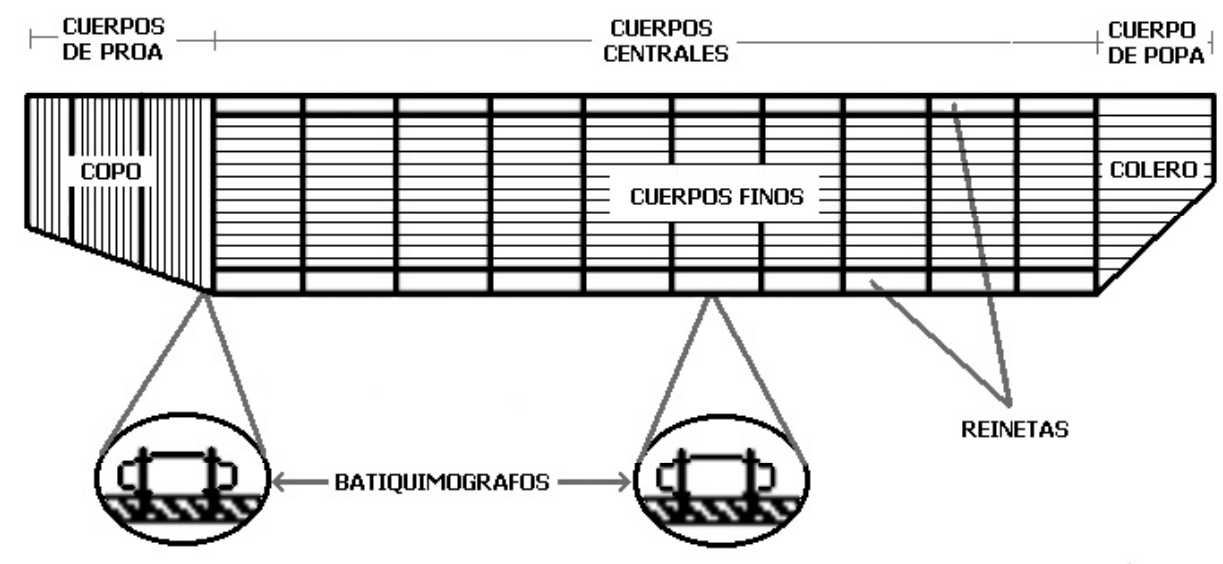

Figura 1. Ubicación de los batiquimógrafos en las redes de cerco durante el estudio.

Figure 1. Batykymographs position on the purse seine net during this research. 
Tabla 1. Características de la tela empleada en las redes de cerco. Table 1. Netting characteristics employed in the purse seine nets.

\begin{tabular}{|l|cc|}
\hline \multirow{2}{*}{ Cuerpo } & \multicolumn{2}{|c|}{ Material, construcción y titulación } \\
\cline { 2 - 3 } & Red 1 & Red 2 \\
\hline \multirow{2}{*}{ Copo } & PA Torcida con nudo & PA Torcida con nudo \\
& $210 \mathrm{~d} / 27-210 \mathrm{~d} / 51$ & $210 \mathrm{~d} / 27-210 \mathrm{~d} / 51$ \\
\hline Centrales & PA Torcida sin nudo & PA Torcida con nudo \\
Copo centro & $210 \mathrm{~d} / 18$ & $210 \mathrm{~d} / 18$ \\
(cuerpo fino central) & PA Torcida sin nudo & PA Torcida con nudo \\
Colero & $210 \mathrm{~d} / 24$ & $210 \mathrm{~d} / 24$ \\
& PA Torcida sin nudo & PA Torcida con nudo \\
\end{tabular}

Tabla 2. Características de las relingas y llave empleadas en las redes de cerco.

Table 2. Float and lead lines and pursing wire characteristics employed in the purse seine nets.

\begin{tabular}{|lcc|}
\hline Estructura & Tipo de material & Diámetro \\
\hline Relinga superior & Polydac-Ultra strong & $1,5 "$ \\
Relinga inferior & Polydac-Ultra strong & $1,5 "$ \\
Llave & Tonina 6x19 & $1,5 ”$ \\
\hline
\end{tabular}

Este análisis estático estuvo referido al cálculo de la altura de tela estirada (HTE), entendiendo ésta como paños centrales sin considerar reinetas, y la estimación del coeficiente de altura $\left(\mu_{2}\right)$ en función del coeficiente de embande (E). A modo comparativo, se calcularon también los pesos lineales sumergidos $\left(\mathrm{kg} \cdot \mathrm{m}^{-1}\right)$ de tela, lastre y total en los cuerpos en estudio (copo y central). La definición de estos cuerpos estuvo en directa relación con su funcionalidad en la red, donde el copo constituyó la sección de embolsamiento de la captura, mientras que el cuerpo central describió el comportamiento vertical del arte que produce el cerco en torno al recurso.

\section{Mediciones in situ}

Las experiencias realizadas en terreno estuvieron referidas a la medición del desempeño operacional de la red, en términos de profundidad de calado y velocidad de hundimiento, utilizando dos batiquimógrafos marca MICREL modelo P2T 600P, dispuestos de igual forma en ambas redes (Fig. 1). Estos sensores permitieron medir y registrar la profundidad en intervalos de tiempo de $5 \mathrm{~s}$ con una precisión de $3 \mathrm{~m}$, siendo esta información almacenada internamente. Esta información fue transferida luego desde cada sensor a un computador personal, utilizando para ello un lector digitalizador y el software SENNE, ambos proporcionados por el fabricante.

Se realizó un total de 21 lances de cerco, correspondientes a 14 lances en la embarcación que utilizó la Red 1 (tela sin nudo en los cuerpos centrales) y 7 lances en la embarcación que utilizó la Red 2 (tela con nudo en los cuerpos centrales). En cada lance, los sensores se ubicaron en dos secciones distintas de la relinga inferior; el primero, se ubicó en la sección del copo (tercer cuerpo de proa de la red), y el segundo, también denominado copo centro, en el cuerpo fino central de la red (noveno cuerpo de la red).

\section{Profundidad de calado (Pc)}

Se determinó la profundidad máxima de calado durante cada lance, definida como el punto más profundo que alcanzó la red $i$ en cada cuerpo $j$ (copo y cuerpo central). Luego, se determinó la profundidad Pc alcanzada por la red en diferenciales de tiempo de $30 \mathrm{~s}$, a partir de lo cual se realizó un ajuste logarítmico de los datos según la ecuación:

$$
P c_{i, j}=B_{O}+B_{1} \times \operatorname{In}(t)
$$

Posteriormente, se calculó el coeficiente de calado (k), definido como la relación entre la profundidad media que alcanzó cada red durante el estudio y la altura de tela estirada de acuerdo a su construcción (Salas et al., 1985), determinándose un indicador del hundimiento o calado en sus cuerpos centrales $(\mathrm{k}=\mathrm{Pc} / \mathrm{HTE})$. 


\section{Velocidad de hundimiento (Vh)}

La velocidad de hundimiento por lance $\mathrm{Vh}$ se determinó a partir del tiempo que tarda cada cuerpo hasta lograr su calado máximo. Luego, se realizó la estimación de $\mathrm{Vh}$ en diferenciales de tiempo de $30 \mathrm{~s}$, procediendo entonces de la siguiente forma:

$$
V h=\frac{\Delta\left(P c_{2}-P c_{1}\right)}{\Delta\left(t_{2}-t_{1}\right)}
$$

Posteriormente, se realizó un ajuste logarítmico de la velocidad Vh alcanzada por la red $i$ en cada cuerpo $j$ en función del tiempo $t$, según la expresión:

$$
V h_{i, j}=B_{O}+B_{1} \times \ln (t)
$$

\section{Comparación de curvas de profundidad y velocidad de hundimiento}

Para la comparación de las curvas ajustadas de profundidad y velocidad de hundimiento, se aplicó el test de pendientes propuesto por Neter et al. (1990), con el fin de determinar estadísticamente las similitudes funcionales entre el desempeño de ambas redes en sus respectivos cuerpos. Las hipótesis planteadas entonces fueron:

$$
\begin{aligned}
& H_{O}: B_{1} \operatorname{red}_{1}=B_{1} \operatorname{red}_{2} \\
& H_{1}: B_{1} \operatorname{red}_{1} \neq B_{1} \operatorname{red}_{2}
\end{aligned}
$$

El estadístico de prueba empleado fue:

$$
t c=\frac{\left(B_{1} \operatorname{Red}_{1}-B_{1} \operatorname{Red}_{2}\right)}{\sqrt{S_{c}^{2} \times\left(\frac{1}{\text { SSXred }_{1}}+\frac{1}{\text { SSXred }_{2}}\right)}}
$$

siendo,

$$
S_{c}^{2}=\frac{\left(\text { SSEred }_{1}+\text { SSEred }_{2}\right)}{n_{1}+n_{2}-4}
$$

donde:

$\mathrm{B}_{1}$ red $_{\mathrm{i}}$ : pendiente de la recta de la red ${ }_{\mathrm{i}}$

$\mathrm{S}_{\mathrm{c}}{ }^{2} \quad$ : varianza combinada de los dos grupos

SSXred $_{\mathrm{i}}$ : suma de cuadrados de X de la red

SSEred $_{i}$ : suma de cuadrados del error de la red $_{i}$

$\mathrm{n}_{\mathrm{i}} \quad$ : número de muestras de la red $_{\mathrm{i}}$

Se comparó el estadístico de prueba $t$ con respecto a un valor crítico $t^{*}$ definido por $t^{*}=t(1-\alpha / 2$; $\left.n_{1}+n_{2}+4\right)$, con $\alpha \operatorname{del} 5 \%$.

\section{RESULTADOS}

\section{Análisis estático}

De la información recopilada para el análisis estático se puede destacar los siguientes aspectos relacionados con las redes en estudio:

\section{Red 1}

En la sección del copo, específicamente el tercer cuerpo de proa, presentó una altura inicial de tela estirada de $100 \mathrm{~m}$ llegando a una altura final de 110 $\mathrm{m}$. Esta última altura es igual a la de los cuerpos centrales y, en ambos casos, su coeficiente de altura es de 0,78 . La longitud total de la relinga superior es de 725,2 m, la sección del copo de 38,6 m y el cuerpo fino central de $54,9 \mathrm{~m}$.

En relación al peso sumergido total calculado, el cuerpo central fue superior en un 58,4\% al cuerpo del copo, con un peso de $583,4 \mathrm{~kg}$. Los principales componentes que determinaron el peso de los cuerpos están representados por el paño y el lastre, siendo para el copo de $23,4 \%$ y $44,0 \%$ del peso total respectivamente. En el cuerpo central estos porcentajes variaron entre $14,5 \%$ y $64,7 \%$. En cuanto a la distribución de pesos por metro de tela, se observó que en el copo, esta relación es de $1,5 \mathrm{~kg} \cdot \mathrm{m}^{-1}, \mathrm{y}$ de $1,3 \mathrm{~kg} \cdot \mathrm{m}^{-1}$ en el cuerpo central. En cambio, el lastre es superior en el cuerpo central con $5,9 \mathrm{~kg} \cdot \mathrm{m}^{-1}$, mientras que en el copo es de $2,9 \mathrm{~kg} \cdot \mathrm{m}^{-1}$ (Tabla 3 ).

\section{Red 2}

El tercer cuerpo de proa presentó una altura inicial de tela estirada de $100 \mathrm{~m}$, aumentando en $19 \mathrm{~m}$ para alcanzar la altura de los cuerpos centrales, equivalente a $119 \mathrm{~m}$; en ambos casos el coeficiente de altura es de 0,75 . La longitud de relinga superior del cuerpo del copo fue de $37,3 \mathrm{~m}$, mientras que el cuerpo fino central tuvo una longitud de 47,5 m. En forma global, la Red 2 tenía una longitud total de relinga superior de $661,4 \mathrm{~m}$.

El peso sumergido total calculado en el cuerpo fino central fue superior en un $90,8 \%$ al cuerpo del copo, con un peso de 747,4 kg. Los principales componentes que determinaron el peso de los cuerpos estuvieron representados por el paño y el lastre, siendo éstos para el copo de $20,6 \%$ y $39,4 \%$ del peso total respectivamente. En el cuerpo central estos porcentajes fueron de $23 \%$ y $50,4 \%$. Se observó que el peso por metro de tela se distribuyó en el copo a razón de $1,5 \mathrm{~kg} \cdot \mathrm{m}^{-1}$, mientras que en el cuerpo cen- 
tral fue de $2,7 \mathrm{~kg} \cdot \mathrm{m}^{-1}$. El lastre fue superior en el cuerpo central con $5,9 \mathrm{~kg} \cdot \mathrm{m}^{-1}$, mientras que en el copo fue de $2,8 \mathrm{~kg} \cdot \mathrm{m}^{-1}$ (Tabla 3 ).

\section{Profundidad de calado}

El registro máximo de profundidad de calado en el tercer cuerpo del copo de la Red 1 fue de 54,7 m, siendo en promedio de 42,0 $\mathrm{m}$ durante los 14 lances realizados. El copo de la Red 2 registró un máximo de $89,5 \mathrm{~m}$, siendo 77,0 $\mathrm{m}$ su profundidad media de calado en los 7 lances ejecutados. En términos de los cuerpos finos centrales, la Red 1 presentó una profundidad máxima de $88,6 \mathrm{~m}$, con un promedio de 71,3 m, mientras que la Red 2 caló hasta un máximo de $105 \mathrm{~m}$, con un promedio de 93,8 m (Tabla 4).

Con respecto a la comparación de las curvas de profundidad de calado del copo, se encontró que el valor de $t_{c}(12,09)$ fue mayor que $t^{*}(2,04)$, por lo cual la hipótesis de nulidad se rechazó, demostrando la existencia de diferencia entre ambas redes. Al comparar esta variable entre los cuerpos centrales, se determinó el mismo efecto, donde el valor de $t$ $(9,02)$ fue mayor que $t^{*}(2,03)$, rechazándose la hipótesis de nulidad, demostrando la existencia de diferencia entre profundidades máximas de calado de los cuerpos centrales de ambas redes (Tabla 5, Fig. 2).

\section{Velocidad de hundimiento}

De acuerdo a los registros de profundidad y tiempo, se determinó la velocidad de calado promedio del copo en los lances muestreados, siendo de 7,5 y 9,8 $\mathrm{m} \cdot \mathrm{min}^{-1}$ para la Red 1 y Red 2, respectivamente. En los cuerpos centrales en cambio, la velocidad de

Tabla 3. Dimensiones, pesos y relaciones lineales de las redes.

Table 3. Nets dimensions, weights and linear relationships.

\begin{tabular}{|c|c|c|c|c|c|}
\hline & & \multicolumn{2}{|c|}{ Red 1} & \multicolumn{2}{|c|}{ Red 2} \\
\hline & & $\begin{array}{l}3^{\text {er }} \text { cuerpo } \\
\text { copo }\end{array}$ & $\begin{array}{c}\text { Cuerpo } \\
\text { fino central }\end{array}$ & $\begin{array}{c}3^{\text {er }} \text { cuerpo } \\
\text { copo }\end{array}$ & $\begin{array}{c}\text { Cuerpo } \\
\text { fino central }\end{array}$ \\
\hline $\begin{array}{l}\text { Dimensiones y } \\
\text { coeficientes }\end{array}$ & $\begin{array}{l}\operatorname{LRS}^{*}(\mathrm{~m}) \\
\operatorname{HTE}^{*}(\mathrm{~m}) \\
\mu_{2}\end{array}$ & $\begin{array}{r}38,6 \\
100-110 \\
0,78\end{array}$ & $\begin{array}{r}54,9 \\
110 \\
0,78\end{array}$ & $\begin{array}{r}37,3 \\
100-119 \\
0,75\end{array}$ & $\begin{array}{r}47,5 \\
119 \\
0,75\end{array}$ \\
\hline $\begin{array}{l}\text { Pesos sumergidos } \\
(\mathrm{kg})\end{array}$ & $\begin{array}{l}\text { Paños } \\
\text { Lastre } \\
\text { Total } \\
\end{array}$ & $\begin{array}{r}86,3 \\
162,1 \\
368,4 \\
\end{array}$ & $\begin{array}{r}84,7 \\
377,6 \\
583,4 \\
\end{array}$ & $\begin{array}{r}80,8 \\
154,2 \\
391,7 \\
\end{array}$ & $\begin{array}{l}171,7 \\
376,4 \\
747,4\end{array}$ \\
\hline $\begin{array}{l}\text { Pesos lineales } \\
\text { sumergidos }\left(\mathrm{kg} \cdot \mathrm{m}^{-1}\right)\end{array}$ & $\begin{array}{l}\text { Paños } \\
\text { Lastre } \\
\text { Total }\end{array}$ & $\begin{array}{l}1,5 \\
2,9 \\
6,5\end{array}$ & $\begin{array}{r}1,3 \\
5,9 \\
9,11\end{array}$ & $\begin{array}{l}1,5 \\
2,8 \\
7,1\end{array}$ & $\begin{array}{r}2,7 \\
5,9 \\
11,7\end{array}$ \\
\hline
\end{tabular}

* LRS: Longitud relinga superior; HTE: Altura tela estirada

Tabla 4. Profundidad de calado y velocidad de hundimiento, valores máximos y desviación estándar.

Table 4. Pursing depth and sinking speed, maximum values and standard deviation.

\begin{tabular}{|ll|rr|rr|}
\hline & \multicolumn{2}{|c|}{ Red 1 } & \multicolumn{2}{c|}{ Red 2 } \\
\hline & & $\begin{array}{c}\mathbf{3}^{\text {er }} \text { cuerpo } \\
\text { copo }\end{array}$ & $\begin{array}{c}\text { Cuerpo } \\
\text { fino central }\end{array}$ & $\begin{array}{c}\text { 3 }^{\text {er }} \text { cuerpo } \\
\text { copo }\end{array}$ & $\begin{array}{c}\text { Cuerpo } \\
\text { fino central }\end{array}$ \\
\hline \multirow{2}{*}{ Profundidad (m) } & Máxima promedio & 42,0 & 71,3 & 77,0 & 93,8 \\
& Desviación estándar & 8,3 & 12,3 & 6,9 & 11,7 \\
& Máxima observada & 54,7 & 88,6 & 89,5 & 105,0 \\
\hline Velocidad $\left(\mathrm{m} \cdot \mathrm{m}^{-1}\right)$ & Media de hundimiento & 7,5 & 6,7 & 9,8 & 7,9 \\
& Desviación estándar & 3,0 & 3,0 & 2,3 & 1,2 \\
& Máxima observada & 11,9 & 12,8 & 13,0 & 9,3 \\
\hline$N^{\text {o de lances }}$ & & 14 & 14 & 7 & 7 \\
\hline
\end{tabular}




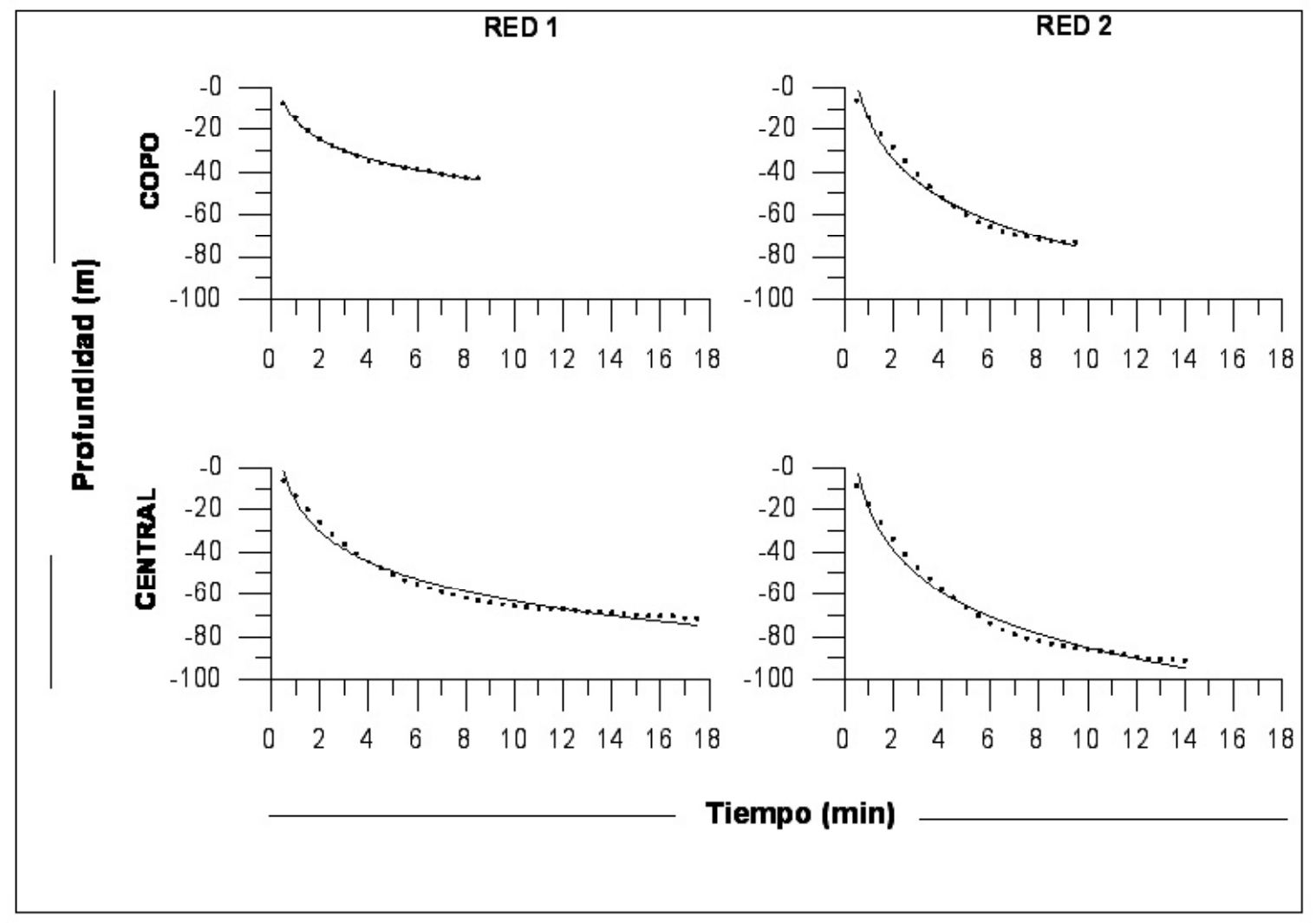

Figura 2. Profundidad de calado en el copo y cuerpo central de las dos redes de cerco en estudio.

Figure 2. Bunt and central body pursing depth of the two research purse seine nets.

hundimiento fue menor, alcanzando a 6,7 y 7,9 $\mathrm{m} \cdot \mathrm{min}^{-1}$ para la Red 1 y Red 2, respectivamente (Tabla 4). En términos diferenciales, los cuerpos del copo en ambas redes presentaron una velocidad de hundimiento entre 12 y $16 \mathrm{~m} \cdot \mathrm{min}^{-1}$ durante los dos primeros minutos de operación, disminuyendo gradualmente hasta una velocidad cero en un lapso promedio de 8 a 10 min desde el calado.

En particular, los cuerpos centrales presentaron diferentes comportamientos entre redes. Se determinó que la Red 1 presentó una velocidad de hundimiento de $15 \mathrm{~m} \cdot \mathrm{min}^{-1}$, disminuyendo en un período de $4 \mathrm{~min}$ a $8 \mathrm{~m} \cdot \mathrm{min}^{-1}$. La Red 2 tuvo una velocidad inicial de $18 \mathrm{~m} \cdot \mathrm{min}^{-1}$, llegando luego de $4 \mathrm{~min}$ a la misma velocidad que la Red 1 , de aproximadamente $8 \mathrm{~m} \cdot \mathrm{min}^{-1}$. El tiempo que tardaron en alcanzar su calado máximo también presentó variación, en un rango de 16-18 min para la Red 1, y 12-15 min para la Red 2.

Al comparar las curvas de velocidad de hundimiento del tercer cuerpo del copo, se observó que el valor de $t_{c}(0,24)$ fue menor que $t^{*}(2,04)$, por lo cual la hipótesis de nulidad se aceptó, existiendo entonces evidencia estadística que avaló la similitud de esta variable entre ambas redes. Un efecto distinto se determinó en la comparación de la velocidad de hundimiento en los cuerpos finos centrales, donde el valor de $t_{c}(4,47)$ fue superior al de $t^{*}$ $(2,01)$, siendo entonces rechazada la hipótesis de nulidad, debido a lo cual se puede afirmar la existencia de diferencia entre sus velocidades de hundimiento (Tabla 6, Fig. 3).

\section{Relación entre profundidad de hundimiento y altura de tela estirada}

De acuerdo a la batimetría alcanzada por los cuerpos centrales y la altura de tela estirada (HTE) de ambas redes se calculó el coeficiente de calado (k), que indicó que la Red 1 logró un hundimiento del $64,9 \%$, mientras que la Red 2 alcanzó un porcentaje superior, equivalente al 78,9\% de HTE. 


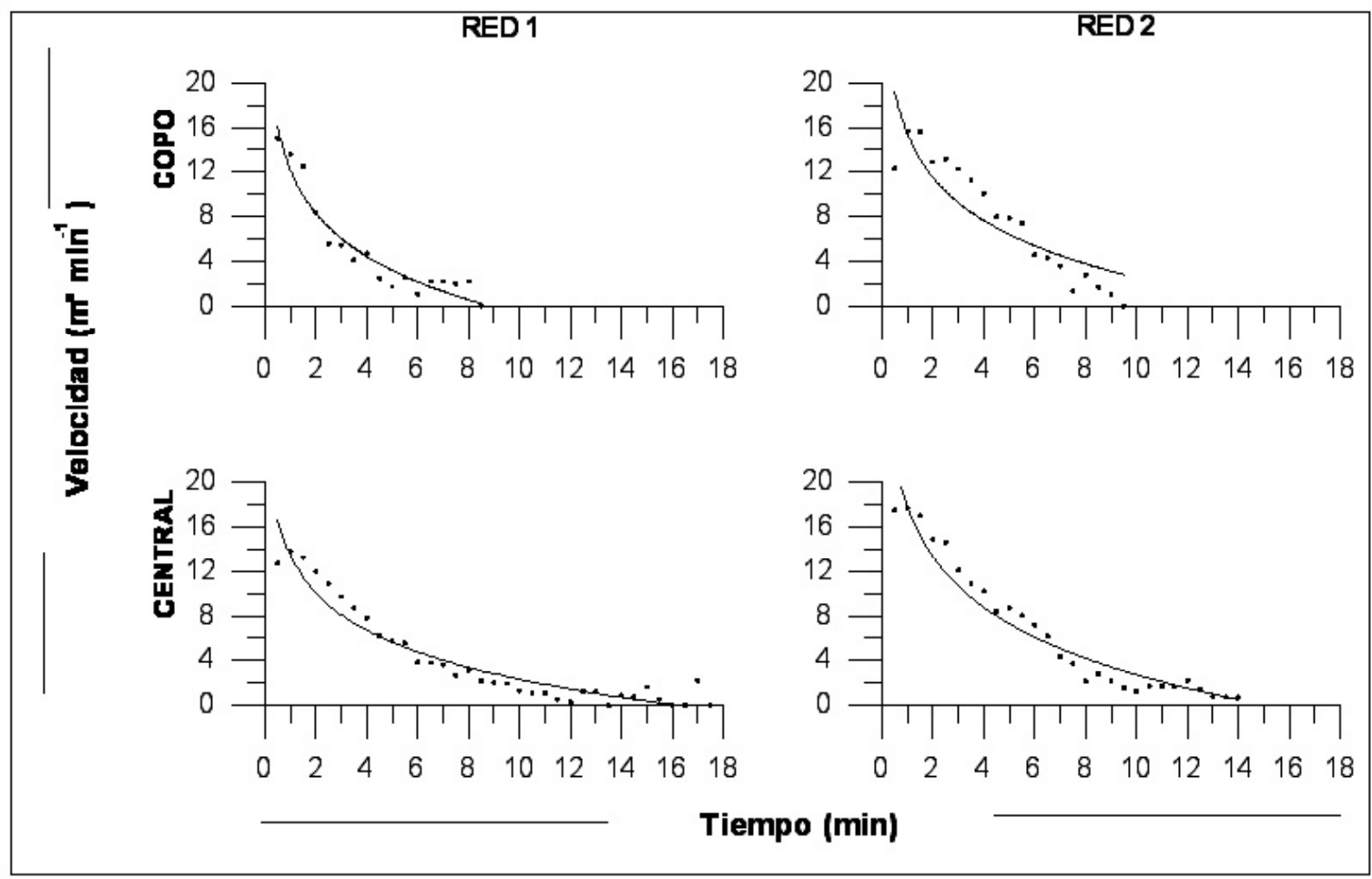

Figura 3. Velocidad de hundimiento en el copo y cuerpo central de las dos redes de cerco en estudio.

Figure 3. Bunt and central body sinking speed of the two research purse seine nets.

\section{DISCUSIÓN}

Las redes empleadas tenían un diseño similar, variando levemente en sus características de armado $\left(\mathrm{m}^{2}\right)$ y, en mayor grado, en la construcción de los cuerpos centrales. Esto último explicaría las diferencias observadas en la velocidad de hundimiento de dichos cuerpos, ya que la Red 1(tela sin nudo) presentó un 22,1\% menos de peso lineal sumergido total, en comparación con la Red 2 (tela con nudo). El origen de esta diferencia radica en el peso lineal sumergido, presentando la Red 1 un 44,4\% menos de tela.

Los cuerpos del copo tenían similares características de construcción, utilizando ambas redes tela con nudo. Se esperaba entonces que el análisis estático demostrase dicha similitud, lo cual se comprobó a través de sus pesos lineales de tela, siendo de $1,5 \mathrm{~kg} \cdot \mathrm{m}^{-1}$ para ambas redes. Esto se observó además, en el peso sumergido del lastre, donde se calculó una diferencia lineal inferior a $0,1 \mathrm{~kg} \cdot \mathrm{m}^{-1}$. El peso lineal total sumergido fue de 2,9 y $2,8 \mathrm{~kg} \cdot \mathrm{m}^{-1}$ para la Red 1 y Red 2, respectivamente, lo que explicó en definitiva la semejanza estadística obser- vada en sus curvas de velocidad de hundimiento.

Si bien existe una diferencia por diseño entre las alturas de tela estirada en los cuerpos centrales equivalente a $9 \mathrm{~m}$, ésta no se reflejó en igual magnitud en las profundidades máxima y media de calado alcanzada por cada una de las redes, ya que en promedio la Red 2 alcanzó 22,5 m más que la Red 1. Este efecto se debería a las diferentes tácticas y maniobras de operación de los patrones de pesca frente al cardumen tal como lo señala Iitaka (1966), quien demostró empleando batiquimógrafos en la red, que un inicio temprano de la maniobra de virado o cierre de la llave reduce la profundidad de calado y, por lo tanto, la eficiencia del cerco.

En este sentido, Mandujano (1998) concluye que las maniobras de calado empleadas en la pesquería de jurel (Trachurus murphyi) en la zona centro-sur de Chile se encuentran relacionadas directamente con una táctica de pesca, indicando que la forma de virado del puntero es una variable netamente operacional que influye sobre el poder de pesca funcional del arte y éste, a su vez, en la captura del recurso. Sin duda, el hecho de no haber considerado va- 
riables de tipo operacional en el presente estudio, tales como la velocidad de las naves durante el calado, velocidades de virado y tiempos de operación, limita en cierto modo el análisis de los resultados, siendo aconsejable registrarlas en estudios posteriores relacionados con el desempeño funcional de las redes de cerco.

El efecto de distribución de pesos lineales en los cuerpos centrales, sumado a variables de operación y distintas tácticas de pesca, posiblemente determinó la diferencia entre las pendientes de las curvas de velocidad de hundimiento. Se observó entonces que la Red 2 cala a mayor velocidad durante los primeros minutos del lance, requiriendo menos tiempo para alcanzar su profundidad máxima en comparación con la Red 1. Sin embargo, es probable que un mayor tiempo de calado en la operación de la Red 1 le hubiese permitido alcanzar mayor profundidad. No obstante, es necesario indicar que las diferencias entre las profundidades alcanzadas pueden ser también un efecto de variables ambientales, particularmente por diferencias en las corrientes marinas, tanto en su velocidad como sentido (Inada et al., 1997; Gaertner \& Sacchi, 2000).

En términos generales, se observó que la máxima velocidad de hundimiento para cada uno de los cuerpos en estudio, se logró durante los dos primeros minutos de operación, lo que concuerda con lo expuesto por Santana et al. (2002) en redes de cerco utilizadas en los océanos Atlántico e Indico. Este corto período de tiempo, es fundamental en el éxito del lance en términos de profundidad de calado y volumen de encierre.

Otro factor importante de señalar, es la presencia o ausencia de nudos en la construcción del paño, ya que Konagaya (1971) y Ben-Yami (1994) señalan que el efecto del nudo influye sobre el arrastre hidrodinámico, disminuyendo la velocidad de hundimiento de la red. Sin embargo, en el presente estudio, no fue posible comparar el efecto de esta variable, ya que además de las diferencias en los paños (con y sin nudo en los cuerpos centrales), existió diferencias en la altura de los cuerpos y su peso lineal. No obstante, y bajo las consideraciones del presente estudio, se puede discutir que la resistencia hidrodinámica por efecto de los nudos en la tela puede ser compensado con un aumento del peso lineal sumergido de la red, lográndose entonces igual o mayor profundidad de calado y velocidad de hundimiento.
Por último, se puede confirmar la validez técnica de utilizar batiquimógrafos para estudiar el desempeño operacional de redes de cerco $y$, a través de esto, determinar su poder de pesca funcional. En efecto, el presente estudio y otros similares, como los realizados por Santana et al. (2002) y Gaertner \& Sacchi (2000) en redes atuneras, evidencian que estos instrumentos son una alternativa simple y relativamente económica, siendo de fácil instalación en la red y sencilla forma de exportar la información registrada, frente a otros equipos tales como sensores hidroacústicos de profundidad.

\section{REFERENCIAS}

Ben-Yami, M. 1994. Purse seining manual. Fishing News Books, Oxford, 406 pp.

Gaertner, D. \& J. Sacchi. 2000. Changes over time net dimensions in French tropical purse 0seiners operating in the Atlantic Ocean. Coll. Vol. Sci. Pap. ICCAT, 51: 571-580.

Green, R.E. 1969. Depth-time sequential analyses of the operation of two California tuna purse seines. Fish. Ind. Res., 5(5): 191-201.

Hester, F.J., D.A. Aasted \& R.E. Green. 1972. Experimental drum seining for wetfish in California. Commer. Fish. Rev., 34(1-2): 23-32.

Iitaka, Y. 1966. Studies on the mechanical characteristics of purse seines (Mimeo). College of Fisheries Navigation, Marine Engineering and Electronics, St John's, Newfoundland, 21 pp.

Inada, H., J. Sekine, H.S. Nemoto, S. Takeuchi, M. Kagoshi, Y. Anzai \& K. Yabuki. 1997. The influence of environmental conditions on fish catches during purse seining operations for skipjack Katsuwonus pelamis and yellowfin Thunnus albacares in the western Tropical Pacific fishing grounds. J. Tokyo Univ. Fish., 83(1-2): 129-138.

Konagaya, T. 1971. Studies on the design of the purse seine. J. Fac. Fish. Prefectural Univ. Mie, 8(3): 209296.

Mandujano, L. 1998. Análisis de desempeño de las embarcaciones de una flota cerquera industrial a través de índices funcionales-operacionales. Tesis de Ingeniería Pesquera. Pontificia Universidad Católica de Valparaíso, Valparaíso, 90 pp. 
Neter, J., W. Wasserman \& M.H. Kutner. 1990. Applied linear statistical models. Irwin Inc., Homewood, 667 pp.

Prado, J. \& F.P. Beirnaert. 1978. Incidence du déroulement de la manoeuvre de la senne sur les performances du filet. ICES C. M., B: 20, 11 pp.

Salas, N., T. Melo \& A. Reyes. 1985. Poder de pesca funcional en embarcaciones de cerco. En: T. Melo (ed.). Estudios en pesquerías chilenas. Escuela de Ciencias del Mar. Pontificia Universidad Católica de Valparaíso, Valparaíso, pp. 123-130.

Santana, J.C., A. Delgado de Molina, J. Ariz, P. Pallarés \& D. Gaertner. 2002. Algunos datos sobre la profundidad que alcanza el arte de cerco en la pesquería atunera tropical. Coll. Vol. Sci. Pap. ICCAT, 54: 157-171.

Recibido: 6 enero 2003; Aceptado: 16 abril 2003 